\title{
Morphological and histological comparative study between lower lateral cartilages of cleft and non-cleft sides in unilateral cleft lip nasal deformity
}

\author{
Saleh MA, ${ }^{a} M D$; Elshahat $A,{ }^{a} M D ;$ Emara $M,{ }^{b} M D ;$ Hussein $H,{ }^{a} M D$; \\ Smith DJ Jr, ${ }^{c} M D$; Awad MA, ${ }^{a} M D$
}

a) Department of Plastic Surgery, Ain Shams University, Cairo, Egypt.

b) Department of Histology, Ain Shams University, Cairo, Egypt.

c) Division of Plastic Surgery, University of South Florida, USA.

\section{Correspondence:}

Ahmed Elshahat, Department of Plastic Surgery, Faculty of Medicine, Ain Shams University. Email: elshahat70@hotmail.com

\begin{abstract}
Correction of cleft lip nasal deformity is an elusive goal. Controversy exists regarding the cause of the deformity and therefore there is a controversy of how to correct the deformity. Extrinsic theory is based on the presence of deformational forces from outside. The intrinsic theory is associated with deficiency of the lower lateral cartilage.

The aim of this study is to compare morphologically and histologically between the lower lateral cartilages of cleft and non cleft sides in patients with unilateral cleft lip nasal deformity.

This study included 16 patients (5-22 years old). They were operated upon to correct unilateral cleft lip nasal deformity. Length, width and thickness of lateral crura of lower lateral cartilages of cleft and non cleft sides were measured. Punch biopsies from the middle part of the caudal ends of lateral crura were taken and sent for histological and immune histochemical studies.

The lateral crus of the cleft side, was significantly wider and shorter than the non-cleft side. There was no significant difference in the chondroblast, chondrocyte and total cellular number in the lower lateral cartilage of the cleft and non cleft sides. There was significantly less glycosaminoglycan content in the ground matrix of the lower lateral cartilage on the cleft side.

To conclude: The deficient content of the ground matrix of the lower lateral cartilage of the cleft side allowed the external deformational force to change the dimensions of the lateral crus. This proves that both the intrinsic and extrinsic factors play a role in deformity. Correction will necessitate both sutures techniques for repositioning and cartilage grafts for augmentation.
\end{abstract}

\section{Introduction:}

The unilateral cleft lip nasal deformity is a functional and aesthetic deformity which is difficult to correct. The residual sign of an excellent lip repair is the persistent cleft lip nasal deformity. Being unilateral makes it more prominent and difficult to camouflage. ${ }^{1}$

Controversy persists as to whether the cleft nasal deformity is secondary to tissue malposition "extrinsic theory" or is associated with tissue deficiency "intrinsic theory". 2
Whereas the extrinsic theory can be explained by the abnormal muscle insertion, ${ }^{3-7}$ the intrinsic theory is based on the hypothesis of alterations in the cells originating from the neural crest. This results in changes in the cellular and matrix components of the alar cartilage. 8-11 $^{-11}$

Suture suspension techniques of the affected alar cartilage to the upper lateral cartilage, the septum, the contralateral alar cartilage, nasal bone and external bolsters can correct the malposition and repair the unilateral cleft lip 
nasal deformity without the need to use cartilage graft if the extrinsic theory is the only factor. ${ }^{12}$ On the other hand the incisional cartilage relocation techniques and cartilage grafting correct tissue deficiency and are advocated by those surgeons who adopt the intrinsic theory. ${ }^{12}$

The controversies regarding the etiology of the unilateral cleft nasal deformity and the need to use cartilage graft versus sutures techniques, prompt us to perform this study. The aim of this work was to compare the lower lateral cartilages of the cleft and non-cleft side in patients with unilateral cleft lip nasal deformity. The comparison will involve morphological dimensions (length, width and thickness), and histological structures.

\section{Patients and methods:}

This study was performed at the Plastic Surgery Department, Ain Shams University Cairo, Egypt and the Plastic Surgery Unit at the Univesity of South Florida, USA. Sixteen patients (who were operated upon to correct unilateral cleft lip nasal deformity) were included in the study. The data of the patients are shown in Table(1). They were investigated through anthropometric measures, histological and immune-histochemical staining.

Table (1): Patients data.

\begin{tabular}{|c|c|c|}
\hline Sex & 14 male & 2 females \\
\hline Lesion & 9 left side & 7 right side \\
\hline Degree of the cleft lip & 14 complete & 2 incomplete \\
\hline Cleft lip/ cleft lip and palate & 4 isolated cleft lip & 12 cleft lip and palate \\
\hline
\end{tabular}

All patients or their guardians signed consent for the surgical procedure, as well as separate consent for their permission to be included in the study. They all approved to have intraoperative measurements of lower lateral cartilages of both sides and to collect cartilage biopsies to be sent to the laboratory for histological and immunohistochemical studies. The patients were selected as randomized control study. All the patients were operated upon at the Plastic Surgery Department at Ain Shams University Hospitals. Each patient served as his own control (non-cleft side was the control).

General endotrachial anesthesia was used in all patients. Unequal V shaped columellar incision was used. The columellar incision was extended to a bilateral infracartilagenous incision. Careful and complete dissection of the lower lateral cartilages ensued. Anthropometric measurements were done during surgery using digital sliding caliber
Figure(1). One millimeter punch biopsies were taken from the middle part of the caudal end of the lower lateral cartilage from both the cleft and non cleft sides. The intended surgery was then carried out.

Anthropometric assessment involved measuring the thickness of the cartilage at the intercrural, middle, and distal portions of both lateral crura. The length was measured from the intercrural point (which was identified on the intercrural peak) to the distal end of the crus. The width was measured at the widest point, perpendicular to the longitudinal axis of the cartilage. All these measures were the same as mentioned by Park et al 1998. Each item was measured three times and an average figure was calculated.

The biopsies taken were preserved and fixed in formaline 4\% solution, mounted in Paraffin blocks and sectioned using microtome. The slides were deparaffinized and rehydrated and stained. Hematoxylin and eosin stains were 
used to show the cellular components. Periodic acid Schiff"s stain was used to show the proteoglycans contents in the ground matrix. Masson`s Trichrome stain was used to show the collagen content of the ground matrix. Toludine blue stain was used to show the sulphated glycosaminoglycan content of the ground matrix.

Quantitative measurements of alar cartilage were achived by using the computerized image analyzer (Leica Qwin 500 image, California, USA), at the Histology Department at Ain Shams University and the National Institute of research in Egypt.

The specimens were stained with hematoxylin and Eosin stains and examined under high power magnification (X20). Counting of the chondroblast, chondrocytes and the total cellular count was done in a fixed surface area $\left(40.9 \mu \mathrm{m}^{2}\right)$ and repeated in five different fields of the specimen and the average number of cells was calculated.

The measurements of the content of the proteoglycans, collagen and sulphated glycosaminoglycans in the ground matrix, was performed using the grey level image. The areas of reactivity were masked and the optical density of special stain reaction was measured. This was done in a fixed surface area (19500 $\mu \mathrm{m}^{2}$ ) and repeated in five different fields of the specimen and the average value was calculated.

The image was transformed into a grey image [a grid of pixels each representing the intensity or brightness at that point by a range of numbers, typically from 0 (black) to 255 (white)]. This means that the lower the readings of the grey level, the higher the contents of glycogen, collagen and sulphated glycosaminoglycans in the ground matrix were and vice versa.

Eight patients were examined by immunehistochemical staining for the presence of the Fibroblast Growth Factor 18 (FGF18) [which promotes chondrogenic cells differentiation (Davidson et al) $]^{13}$ and collagen type I and II [the alar cartilages have high density of type II collagen but almost none of type I (Popko et al). ${ }^{14}$

The Santa Cruz Biotechnology Antigen Antibody Complex (ABC) goat staining system was used to detect the FGF18 in the cleft and non-cleft side specimens while the Santa Cruz Biotechnology Antigen Antibody Complex (ABC) rabbit staining system was used to detect the collagen I and II in the lower lateral cartilage of both cleft and non-cleft side specimens.

Objective analysis using the Bioquant Nova Prime Core/Satellite System version 6.90.10, Bioquant Image Analysis Corp., Nashville, TN, USA was used to examine the immune histochemical stains in the cleft and non-cleft lower lateral cartilage. This was carried out on the Plastic Surgery Unit, at the University of South Florida, USA.

Specimens stained with Santa Cruz Biotechnology ABC goat staining system used to detect the FGF18 in the cleft and non-cleft side specimens were examined under high power magnification (X20) using the Leica Qwin 500, California, USA. Counting the chondrocytes was done in a fixed surface area $\left(40.9 \mu \mathrm{m}^{2}\right)$ and it was repeated in three different fields of the specimen and the average number of cells was calculated in the cleft and noncleft alar cartilage. The ratio of the stained cells with FGF18 to the non stained cells was calculated.

Anthropometric and histologic data were analysed statistically by a medical statistician using SPSS package version 15 for the Paired T-test. Immune histochemistry data were analysed statistically using Sigma Stat 3.5 (for Windows), Systat Software, Inc, Point Richmond, CA, USA for the Wilcoxon signed rank sum test.

\section{Results:}

\section{Anthropometric results:}

The lower lateral cartilage at cleft side was shorter than the non cleft side $[\mathrm{P}<0.03$ (statistically significant)], Figure(2).

The lower lateral cartilage at cleft side was wider than the non cleft side $[\mathrm{P}<0.02$ (statistically significant)], Figure(3).

The lower lateral cartilage at cleft side was thinner than the non cleft side $[\mathrm{P}=0.05$ (non significant but shows a trend)].

\section{Histological results:}

There was no statistically significant difference in the chondroblast, chondrocyte 
and total cellular number in the lower lateral cartilage of the cleft and non cleft sides, Figure(4).

There was no statistically significant difference in the grey level image of the Masson Trichrome Stain (no significant difference in collagen content in the ground matrix of the lower lateral cartilage at the cleft and the noncleft sides), Figure(5).

There was no statistically significant difference in the grey level image of the Periodic Acid Schiff Stain (no significant difference in proteoglycans content in the ground matrix of the lower lateral cartilage at the cleft and the non-cleft sides), Figure(6).

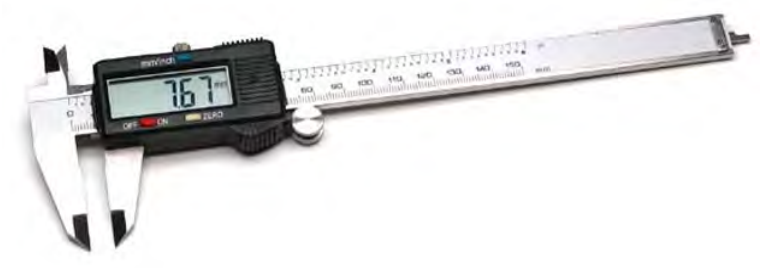

Figure (1): Shows the digital sliding caliber.

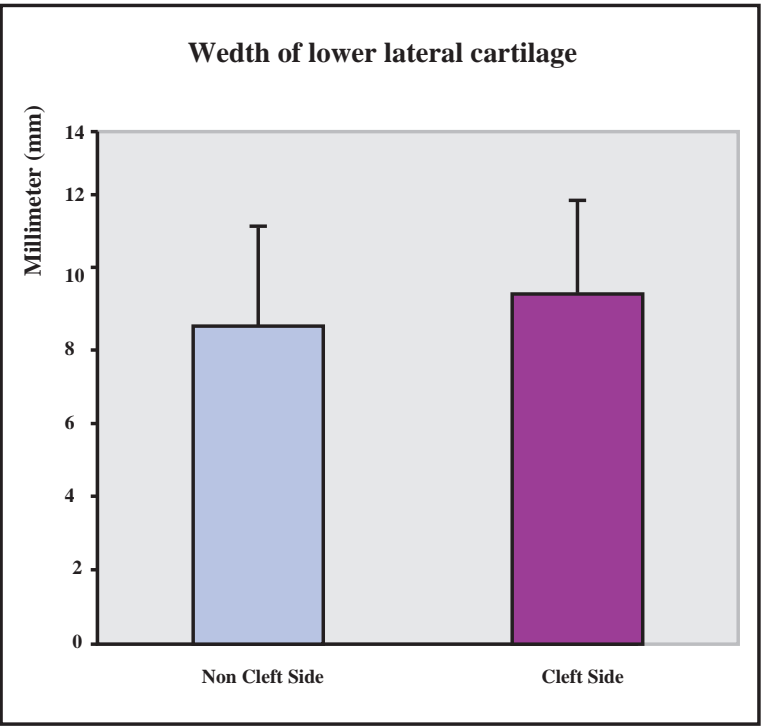

There was statistically significant difference in the grey level image of the Toluidine Blue Stain [The glycosaminoglycan (GAGs) content in the ground matrix of the lower lateral cartilage at the cleft side was significantly less than that of the non-cleft side $(\mathrm{P}<0.01)$ ],

\section{Figures(7,8).}

\section{Immune histochemistry results:}

The data did not follow a normal distribution and therefore, Wilcoxon Signed Rank Sum test was used (a non-parametric method that is corresponding to the Paired t-test). There was no significant difference in the ratio of stained/ non stained cell in the cleft and non cleft side $(\mathrm{P}=0.5186)$, Figure(9).

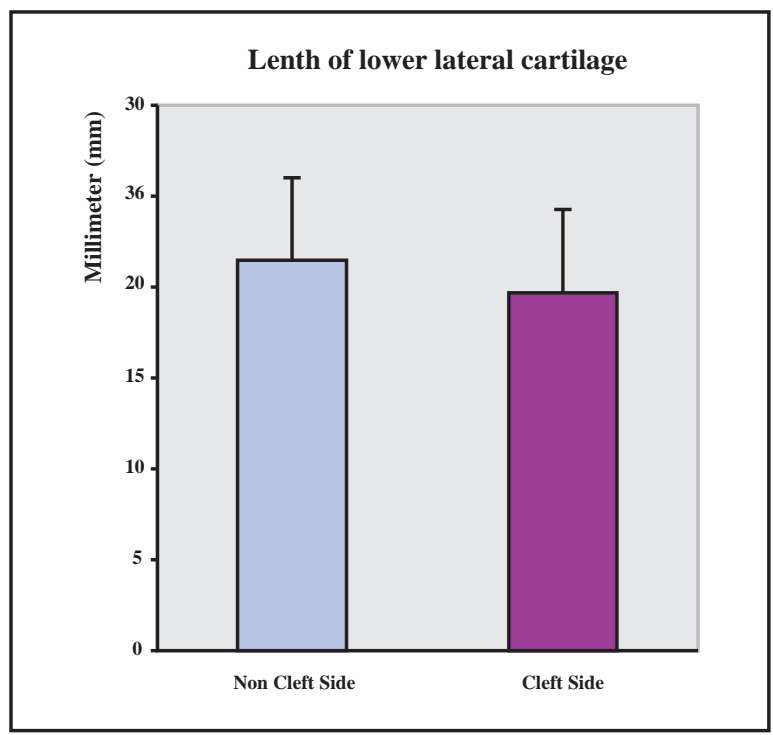

Figure (2): The diagram shows the difference between the length of the lower lateral cartilage in the cleft and non cleft side.
Figure (3): The diagram shows the difference between the width of the lower lateral cartilage in the cleft and non cleft side. 

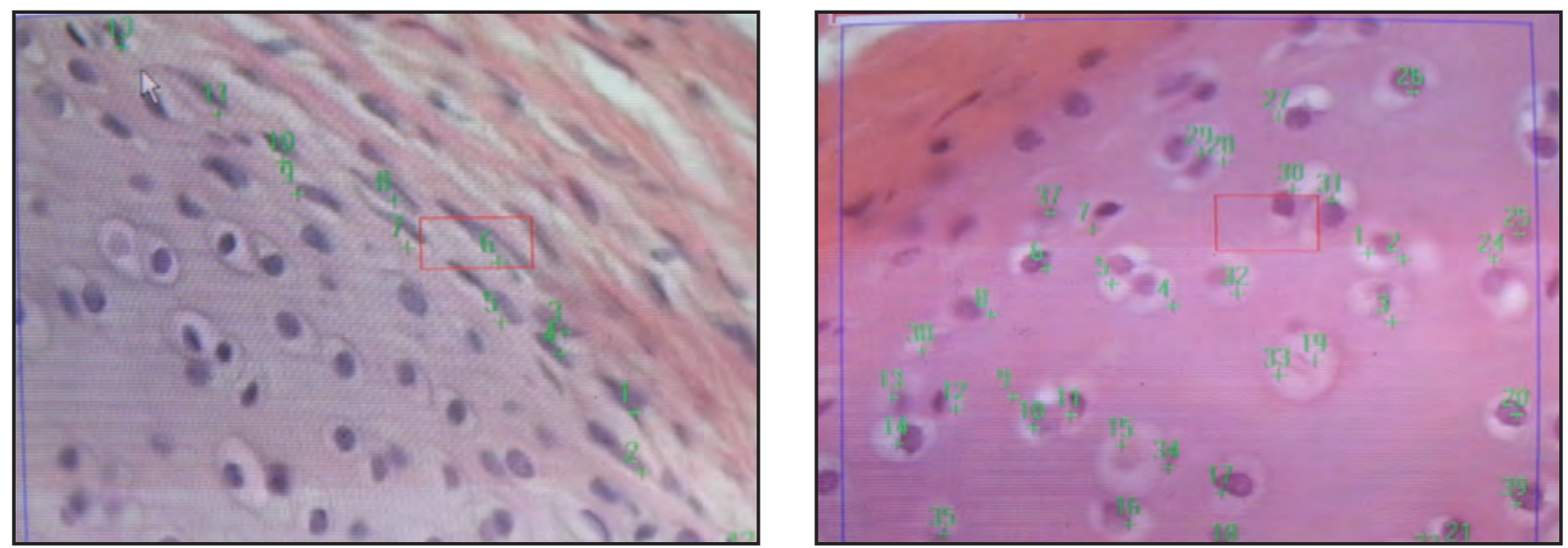

Figure (4): Specimens stained with Hematoxylin and Eosin were examined under high power magnification (X 20); (left specimen) counting the chondroblast; (Right Specimen) counting the chondrocytes.

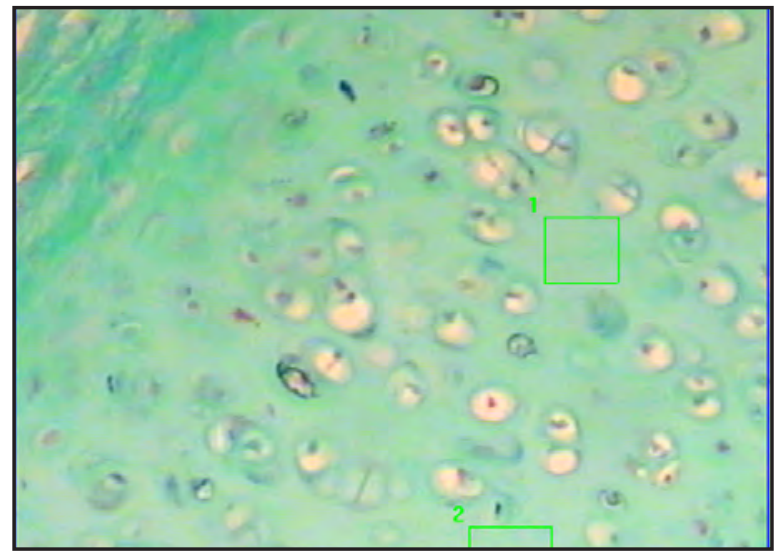

Figure (5): The specimen is stained with Masson's trichrome stain that shows the collagen content of the ground matrix.

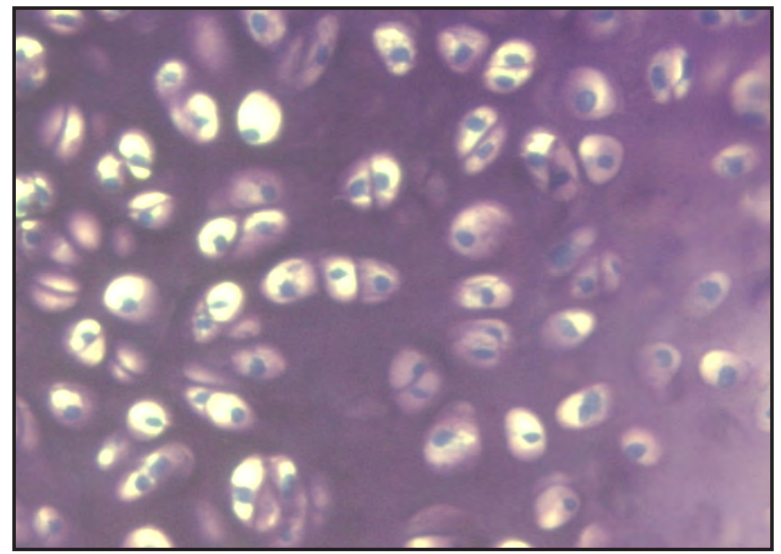

Figure (7): The specimen is stained with Toluiden blue stain that shows the glycosaminoglycans content of the ground matrix (20 x magnifications).

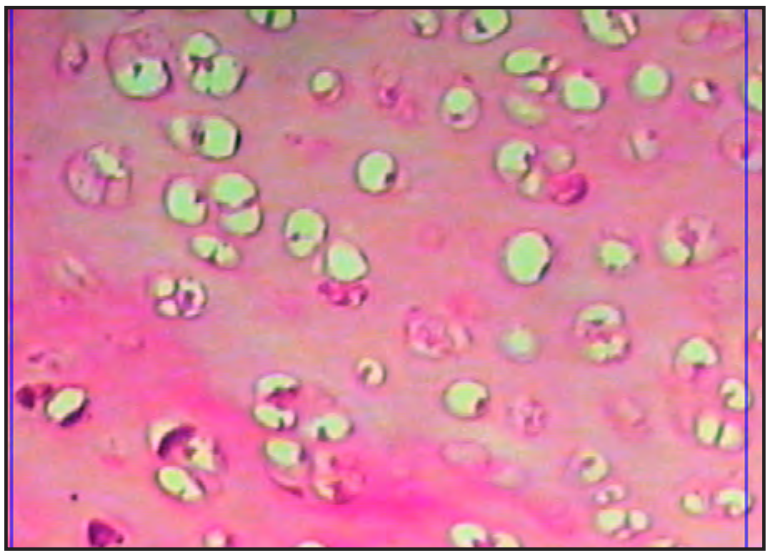

Figure (6): The specimen is stained with periodic acid Schiff stain that shows the proteoglycans content of the ground matrix.

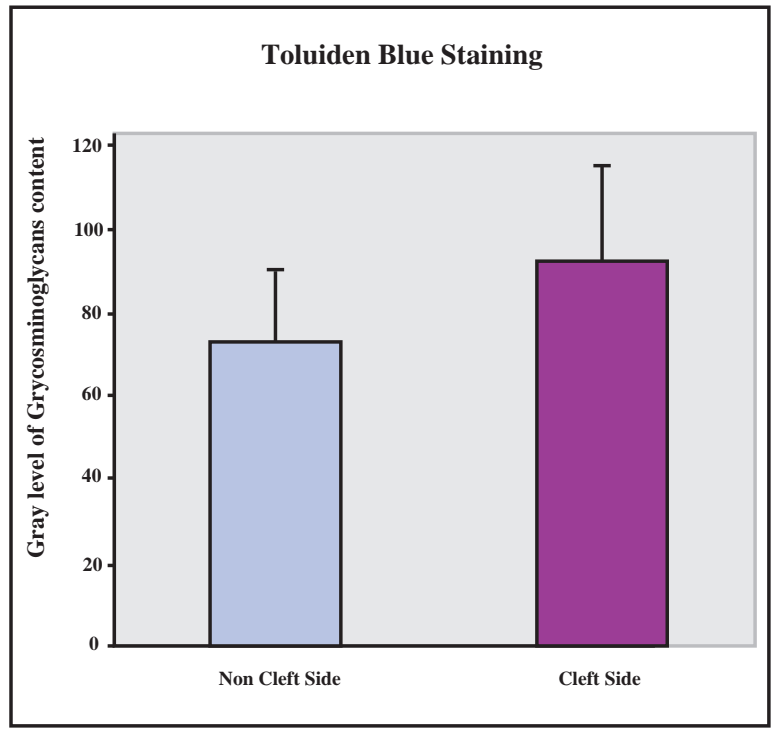

Figure (8): The diagram shows the difference between the glycosaminoglycans content in the ground matrix of the lower lateral cartilage in the cleft and non cleft side. 

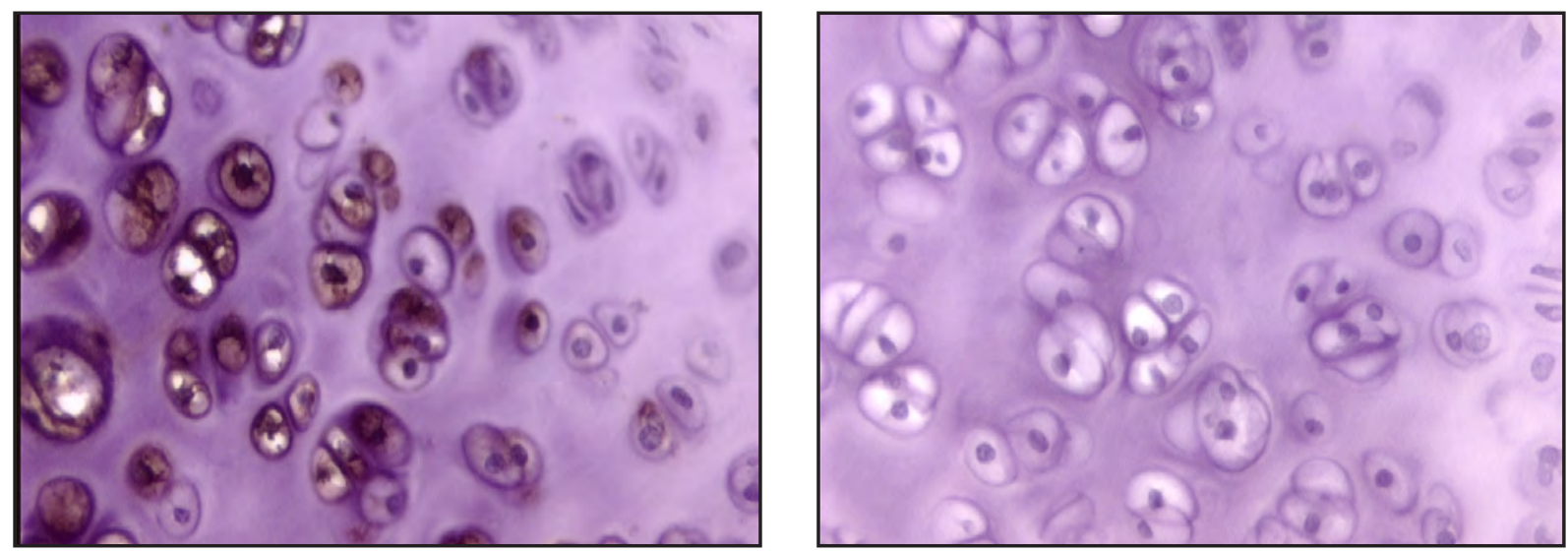

Figure (9): The left specimen shows cells stained with FGF18 antibodies (Brown cells) and the cells which are not stained. The right specimen is the control slide.

\section{Discussion:}

The pathogenesis of the cleft lip nasal deformity has been investigated for a long time and still there is a dilemma as regard this unique complex deformity. It is well known from previous studies that the lower lateral cartilage at the cleft side has specific deformity due to the external force of the misdirected perinasal and orbicularis oris muscles. $3,4,6,7,11,15-17$

Our main concern was to investigate whether the lower lateral cartilage was hypoplastic as mentioned by Avery, ${ }^{10}$ Tulenko, ${ }^{18}$ and Spira et al. ${ }^{19}$ If it is hypoplastic (either in size, content or both) this makes it more susceptible to the external deformational forces. ${ }^{2,20-27}$

The dimensions of the lower lateral cartilage taken using a digital sliding caliber are more accurate $(0.01 \mathrm{~mm})$ than the liebinger sliding caliber used by Park et al. ${ }^{6}$

This current study showed broader and shorter lower lateral cartilage on the cleft side. It showed a trend to be thinner than that on the non cleft side. This differs from the results of Park et al, 16 which showed wider and thicker lower lateral cartilage on cleft side with similar length. This difference may be explained by the difference in external deformational forces which depend on the difference in surgical technique of primary cleft repair.

Punch biopsy technique to harvest cartilage for histological and immune histochemical staining was previously mentioned by Park et $\mathrm{al}^{6}$ and Sadove et al. ${ }^{28}$ However, Sadove et $\mathrm{al}^{28}$ biopsies were taken from the midportion of the medial crura which is not the main site for the pathology. Spira et al ${ }^{19}$ reported that hypoplasia of the lateral crus of alar cartilage was the primary cause in unilateral cleft lip nasal deformity.

The results of this study concord with that of Park et al ${ }^{6}$ and Modolin et al. ${ }^{11}$ There was non- significant difference between the cleft and non cleft sides cellular count. However, there was a significantly less glycosaminoglycan content in the ground matrix of the lower lateral cartilage in the cleft side. This may explain why the lower lateral cartilage is more susceptible to the external deformational force and became wider and thinner. On the other hand, this glycosaminoglycan deficiency can be a reaction to excessive traction.

Non-significant difference between the cleft and non cleft sides regarding the ratio of the stained cells with FGF18 to the non stained cells indicated that there was no difference in chondrogenic cell differentiation.

We can conclude from the current study that the pathogenesis of the cleft lip nasal deformity is not only due to extrinsic factor which is not a debatable subject but also due to an intrinsic deficiency of the lower lateral cartilage. Deficient glycosaminoglycan of the ground matrix of the lower lateral cartilage on the cleft side makes it prone to the deformational forces by the malinserted perinasal and orbicularis oris muscle. Both suture suspension techniques and cartilage grafting should be used together to correct cleft lip nasal deformity. 


\section{References:}

1- Sykes JM, Senders CW: Surgical treatment of unilateral lip nose deformity at the time of lip repair. Fac Plast Surg Clin North Am 1995; 3: 69.

2- Byrd HS, El-Musa KA, Yazdani A: Definitive repair of the unilateral cleft lip nasal deformity. Plast Reconstr Surg 2007; 120: 1348.

3- Huffman W, Lierle D: Studies in the pathological anatomy of the unilateral harelip. Plast Reconstr Surg 1949; 4: 225.

4- Stenstrom SJ, Oberg T: The nasal deformity in unilateral cleft lip. Plast Reconstr Surg 1961; 28: 295.

5- Novoselov RD: Mechanism of deformities of the middle part of the face occurring by inborn clefts unilateral. Acta Chir Plast 1979; 21: 7.

6- Park B, Lew D, Lee Y: A comparative study of the lateral crus of alar cartilage in unilateral cleft lip nasal deformity. Plast Reconstr Surg 1998; 101: 915.

7- Li AQ, Sun YG, Wang GH, Zhong ZK, Cutting C: Anatomy of the nasal cartilage of the unilateral complete cleft lip nose. Plast Reconstr Surg 2002; 109: 1835.

8- Patten BM: Embryology of the palate and the maxillofacial region. In: Cleft lip and palate. Grabb WG, Rosenstein SW, Bzoch KR (Editors); Boston: Little, Brown (Publisher);1971.

9- Stark RB, Kaplan JM: Development of the cleft lip nose. Plast Reconstr Surg 1973; 51: 413.

10-Avery JK: The evolution of its surgery. In: Cleft craft. Millard RD (Editor); Boston: Little, Brown (Publisher); 1976.

11- Modolin M, Baracat G, Kamakura L, Cintra JrW, Cruz L, Ferreira M: Histological comparison of the alar nasal cartilages in unilateral cleft lip. Rev Hosp Clin Fac Med Sao Paulo 2002; 57: 143.

12-Madorsky SJ, Wang TD: Unilateral cleft rhinoplasty: a review. Otolaryngol Clin North Am 1999; 32: 669.

13-Davidson D, Blane A, Filion D, Wang H, Plut P, Pfeffer G: Fibroblast growth factor (FGF) 18 signals through FGF receptor 3 to promote chondrogenesis. $\mathrm{J} \mathrm{Biol} \mathrm{Chem}$ 2005; 280: 20509.

14-Popko M, Bleys L, De Groot W, Huizing $\mathrm{H}$ : Histological structure of the nasal cartilages and their perichondrial envelope. I- the septal and lobular cartilage. Rhinology 2007; 45: 148.

15-Fara M: The musculature of cleft lip and palate. In: Reconstructive Plastic Surgery. Converse JG, McCarthy JG (Editors). Philadelphia: Saunders (Publisher); 1977.

16-Heckler FR, Oesterle LG, Jabaley ME: The minimal cleft lip revisited: Clinical and anatomical correlations. Cleft Palate J 1979; 16: 240.

17-Green MF: The embryological development and functional importance in the repair of the nasal mucosa to reduce the deformity of the cleft lip nose. Scand J Plast Reconstr Surg 1987; 21: 1.

18-Tulenko JF: Cleft lip nasal deformity in the absence of cleft lip: case report. Plast Reconstr Surg 1968; 41: 35.

19-Spira M, Hardy SB, Gerow FJ: Correction of nasal deformities accompanying unilateral cleft lip. Cleft Palate J 1970; $7: 112$

20-Reynolds JR, Horton CE: An alar lift procedure in cleft lip rhinoplasty. Plast Reconstr Surg 1965; 35: 377.

21-Berkeley WT: Correction of secondary cleft-lip nasal deformities. Plast Reconstr Surg 1969; 44: 234.

22-Whitlow DR, Constable JD: Crossed alar wing peocedure for correction of late deformity in the unilateral cleft lip nose. Plast Reconstr Surg 1973; 52: 38.

23-Malek R: Nasal deformities and their treatment in secondary repair of cleft lip patients: A clinical study of 175 cases. Scand J Plast Reconstr Surg 1974; 8: 136.

24-McComb H: Treatment of the unilateral cleft lip nose. Plast Reconstr Surg 1975; 55: 596.

25-Sawhney CP: Nasal deformity in unilateral cleft lip. Cleft Palate J 1976; 13: 291.

26-Millicovsky G, Ambrose LH, Johnston MC: Developmental alteration associated with spontaneous cleft lip and palate in CL/FR mice. Am J Anatomy 1982; 164: 29.

27-Rifley W, Thaller S: The residual cleft lip nasal deformity: What's new in plastic surgery? Clin Plast Surg 1996; 23: 81.

28-Sadove R, Ladaga L, Magee WP: Cartilaginous histology of the cleft lip nose: Proving the extrinsic etiology. Plast Reconstr Surg 1988; 8: 655. 